\title{
Monitoring the late decline of the old nova RW Ursae Minoris
}

\author{
F. Tamburini ${ }^{1}$, F. Di Mille ${ }^{1}$, A. Bianchini ${ }^{1,2}$, and P. Johnson ${ }^{2}$ \\ 1 Department of Astronomy, University of Padova, vicolo dell'Osservatorio 2, 35122 Padova, Italy \\ e-mail: antonio.bianchini@unipd.it \\ 2 Department of Physics and Astronomy, University of Wyoming, Laramie, WY 82071, USA
}

Received 7 November 2006 / Accepted 18 December 2006

\section{ABSTRACT}

\begin{abstract}
New 2003-2006 time-resolved photometric observations of the old nova RW UMi (1956) confirm the QPO nature of the light modulations observed. The analysis of all our historical runs show that QPOs tend to cluster around frequencies $4.96 \mathrm{~d}^{-1}$ and $12.4 \mathrm{~d}^{-1}$ while the historical superhump period remains undetected in our data. Although the two main frequencies observed might appear to be aliases of the superhump frequency $17 \mathrm{~d}^{-1}$, they should be produced by two independent mechanisms. These rather irregular QPOs might be explained by vertical oscillations of the inner regions of the accretion disk and/or by the cyclical generation in the disk of travelling density waves at the co-rotation radius of an inclined magnetic rotator. We find that the post-nova luminosity is decaying at a rate of $\sim 0.03 \mathrm{mag}^{-1}$. If RW UMi is an intermediate polar system, during the decline the magnetospheric radius of the accreting white dwarf primary should increase and produce important changes in the periods of QPOs.
\end{abstract}

Key words. stars: novae, cataclysmic variables - accretion, accretion disks

\section{Introduction}

RW UMi is a slow nova with an unusually large outburst amplitude as it reached maximum luminosity $V \sim 6$ (Kukarkin 1962) while its magnitude before the 1956 outburst was $\sim 21$ (Duerbeck 1987). Since the luminosity of the post-nova is still about 2 mag brighter than the pre-nova and its orbital period is suspected to be below the $2-3 \mathrm{~h}$ period gap of Cataclysmic Variables (CVs) (see Warner 1995, for a comprehensive review on CVs), RW UMi was suggested by Retter \& Lipkin (2001, hereafter RL) to be a non-magnetic $\mathrm{CV}$ that is experiencing a long post-outburst superhump phase like two other short-orbital-period old novae: CP Pup (Patterson \& Warner 1998) and V1974 Cyg (Retter et al. 1997). Typically, superhumps characterise SU UMa DN systems during superoutbursts and are believed to be produced at relatively large $\dot{M}_{2}$, when the outer edge of the accretion disk expands reaching the 3:1 resonance radius. Superhumps then represent the beat period between the apsidal prograde precession of the eccentric outer edge of the disk and the orbital motion of the secondary. For this reason, superhump periods are only a few percent longer than the orbital periods.

The pionnering extensive photometric survey performed by RL revealed a stable period of $0.05912 \pm 0.00015 \mathrm{~d}(\sim 1.42 \mathrm{~h})$ (hereafter PRL) with a 0.025 mag amplitude interpreted as either due to superhumps or to orbital motion. The 1999-2002 photometric observations carried out by Bianchini et al. (2003, Paper I) suggested the presence of a dominant quasi periodic modulation, labelled $P_{0}$, peaking between frequencies 9 and $14 \mathrm{~d}^{-1}$, corresponding to periods in the range $1.7-2.4 \mathrm{~h}$, i.e. about $45 \%$ longer than period PRL which, instead, was not clearly detected. This result was tentatively explained as due to the presence and predominance of low frequency QPOs of the order of that of the orbital period, eventually produced by vertical oscillations of the inner disk region excited by the rotating magnetosphere of the WD. For these reasons, RW UMi was suggested to be an intermediate polar system. Paper I also reported the occasional presence of longer time scale modulations at frequency $\sim 4 \mathrm{~d}^{-1}$ as well as of higher frequency QPOs around 20 and $28 \mathrm{~d}^{-1}$.

In Paper I it was also shown that the mean luminosity of the post-nova is decaying at a rate of $\sim 0.02 \mathrm{mag}^{-1}$. According to Schreiber \& Gänsicke (2001) such a rate of decline could be well explained as the disk being irradiated by the slowly cooling WD. However, the presence of superhumps suggests that the post nova is experiencing a high $\dot{M}_{2}$, produced by the irradiated secondary. Thus, a secular decrease of the mass transfer rate is plausible.

In this paper we present the results of new photometric observations of the old nova. In Sect. 2 we present the observations and the data reduction. The light modulations detected and the long-term light curve of the post nova are presented in Sect. 3. Conclusions are given in Sect. 4.

\section{The observations and data reduction}

RW UMi was observed using the $1.82 \mathrm{~m} \mathrm{Mt}$. Ekar telescope with the CCD of the AFOSC system as an imager. Time resolved observations in $V$ were obtained in 2003 on Feb. 21 and in 2004 on Feb. 16 and 17 (runs E6, E7, and E8, respectively), and in 2006 on May 5, Jun. 26 and Jun. 27 (runs E13, E14 and E15, respectively). Four individual $V$ frames were obtained on Mar. 29 and 30, 2006 labelled as E9, E10, E11 and E12. Comparison stars were chosen in the field as described in Paper I. Data reductions were performed using standard aperture photometry procedure. The journal of the observations in Table 1 reports the starting time (JD), the filter used, the duration $\Delta T$ of each night, the time resolution $\delta T$ of each run or single frame, the magnitude of individual observations or the mean magnitude of the runs, and the estimate of the maximum amplitude of the oscillations observed in the runs. Run E14 was obtained in white light and its mean magnitude has been set to 19.0. Figure 1 shows the light curves 
Table 1. The journal of the observations.

\begin{tabular}{cccccccc}
\hline \hline run $^{a}$ & Date & $\begin{array}{c}\text { JD } \\
\text { start }\end{array}$ & Filter & $\begin{array}{c}\Delta T \\
(\min )\end{array}$ & $\begin{array}{c}\delta T \\
(\mathrm{~min})\end{array}$ & mag & $\Delta$ mag \\
\hline E6 & Feb. 21 2003 & 2452692.381 & $V$ & 349.2 & 1.3 & 19.06 & 0.12 \\
E7 & Feb. 16 2004 & 2453052.262 & $V$ & 236.4 & 5.3 & 18.86 & 0.12 \\
E8 & Feb. 17 2004 & 2453053.384 & $V$ & 497.4 & 5.3 & 18.84 & 0.12 \\
E9 & Mar. 29 2006 & 2453824.472 & $V$ & 1 & 1 & 18.91 & - \\
E10 & Mar. 29 2006 & 2453824.475 & $V$ & 1 & 1 & 18.85 & - \\
E11 & Mar. 30 2006 & 2453825.457 & $V$ & 1 & 1 & 19.09 & - \\
E12 & Mar. 30 2006 & 2453825.497 & $V$ & 1 & 1 & 18.71 & - \\
E13 & May 05 2006 & 2453859.354 & $V$ & 69.6 & 1.3 & 19.1 & 0.10 \\
E14 & Jun. 26 2006 & 2453914.373 & clear & 189 & 1.3 & 19.0 & 0.10 \\
E15 & Jun. 27 2006 & 2453915.444 & $V$ & 102.5 & 1.3 & 18.95 & 0.10 \\
\hline
\end{tabular}

${ }^{a}$ E9-E12 refer to single $V$ frames.

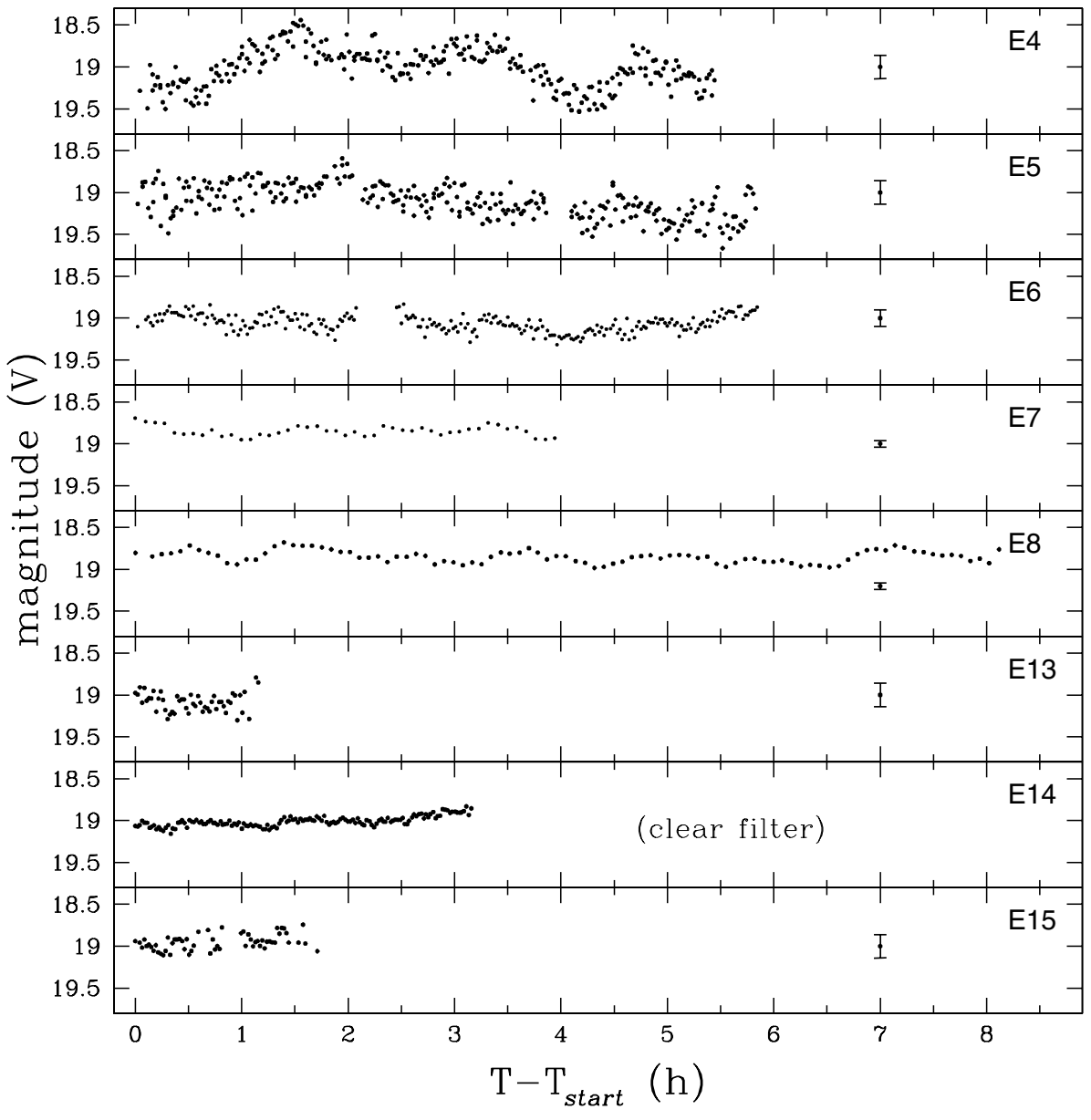

Fig. 1. The light curves of new runs E6, E7, E8, E13, E14 and E15 are shown together with old runs E4 and E5. Typical error bars of individual points are shown. Runs E13 and E15 are rather short due to variable weather conditions. We notice a decrease with time of the amplitude of the light modulations. of new runs E6, E7, E8 and E13 compared to older Ekar runs E4 and E5.

\section{Results}

\subsection{The observed light modulations}

The discrete Fourier transforms (DFT, Deeming 1975) of runs E6, E7, E8 and E14 are shown in Fig. 2. No power at the superhump period PRL is detected and almost no signal is even seen at $P_{0}\left(\sim 10 \mathrm{~d}^{-1}\right)$, the apparently more stable peak discussed in Paper I. If frequencies 4, 9 and $22 \mathrm{~d}^{-1}$ observed in run E6 might be reminiscent of those found in Paper I, the other runs show peaks around frequencies 3-4, 13-14, 21-22 and, perhaps, 29-30 $\mathrm{d}^{-1}$. Run E8 shows two frequencies, $P_{3.1}$ and $P_{13.5}$, that could be aliases of the presumed superhump frequency $17 \mathrm{~d}^{-1}$. However, none of them can be the beat frequency produced by the other and the orbital period because each signal survives when the other one is subtracted from the data. An example is given in the middle-right panel of Fig. 2. The power spectrum of combined runs E7 and E8 shows 1-day aliases and is dominated by the longest run E8. The power spectrum of all our historical runs, carried out with the Wyoming Infrared Observatory (WIRO) and Ekar telescopes (Paper I and this paper), corrected for long-term trends, is shown in Fig. 3. In the upper panel, we notice the presence of only two broad maxima. The peak of the lower frequency maximum is found between the two 1-day aliases at frequencies 4 and $5 \mathrm{~d}^{-1}$, labelled $P_{4}$ and $P_{5}$, respectively. The higher frequency maximum, labelled $P_{12}$, is centered around frequency $12 \mathrm{~d}^{-1}$. Actually, the two broad maxima of the 


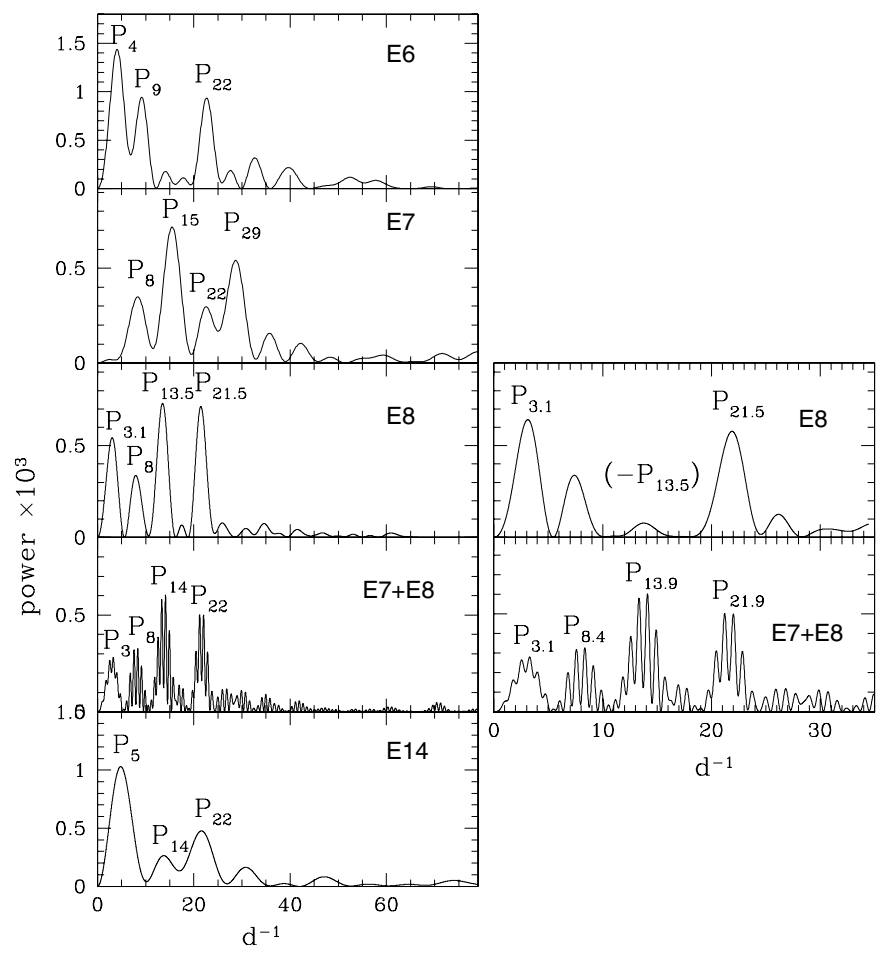

Fig. 2. The power spectra of runs E6, E7, E8 and E14. Main signals fall in the range $3-22 \mathrm{~d}^{-1}$ but peak frequencies change from run to run. None of the two frequencies $P_{3.1}$ and $P_{13.5}$ detected in run E8 can be considered as the beat between the presumed orbital frequency $\sim 17 \mathrm{~d}^{-1}$ because each signal survives to the subtraction of the other one from the data (see example in the middle right panel). The modulations that are more stable over longer time scales are suggested by the power spectrum of all the data in Fig. 3.

power spectrum cannot indicate any strict periodicity. Similarly to what we found in the power spectrum of run E8, also these two peaks might appear to be aliases of the frequency $17 \mathrm{~d}^{-1}$ corresponding to the superhump period PRL, i.e. the orbital period. However, as shown in the middle and lower panel of Fig. 3, each one of these two peaks survives when the other is subtracted from the data. The data folded with the two frequencies $P_{5}$ and $P_{12}$ are shown in Fig. 4. The modulation at frequency $P_{12}$ has a smaller amplitude but is definitely better defined as the folded data points show a smaller dispersion than $P_{5}$.

\subsection{The post-outburst secular light decline}

The post-outburst historical light curve of the nova is represented in Fig. 5. All data points previous to the observations listed in Table 1 are discussed in Paper I. Vertical bars represent the maximum amplitude of the oscillations about the mean magnitude of the time-resolved photometric runs (filled circles). The secular light decline of the old nova, suggested in Fig. 5 by the dashed line that interpolates filled circles, is $\sim 0.03 \mathrm{mag}^{-1}$, that is slightly larger than the value derived in Paper I. The decline is characterized by the presence of oscillations with time scales of a few years.

\section{Discussion}

In Paper I it was suggested that the detection of a dominant photometric modulation $P_{0} \sim 2 \mathrm{~h}$, instead of the historical superhump period $P_{\mathrm{RL}} \sim 1.42 \mathrm{~h}$, could be ascribed to the presence of low frequency QPOs that affect or even dominate the light curve

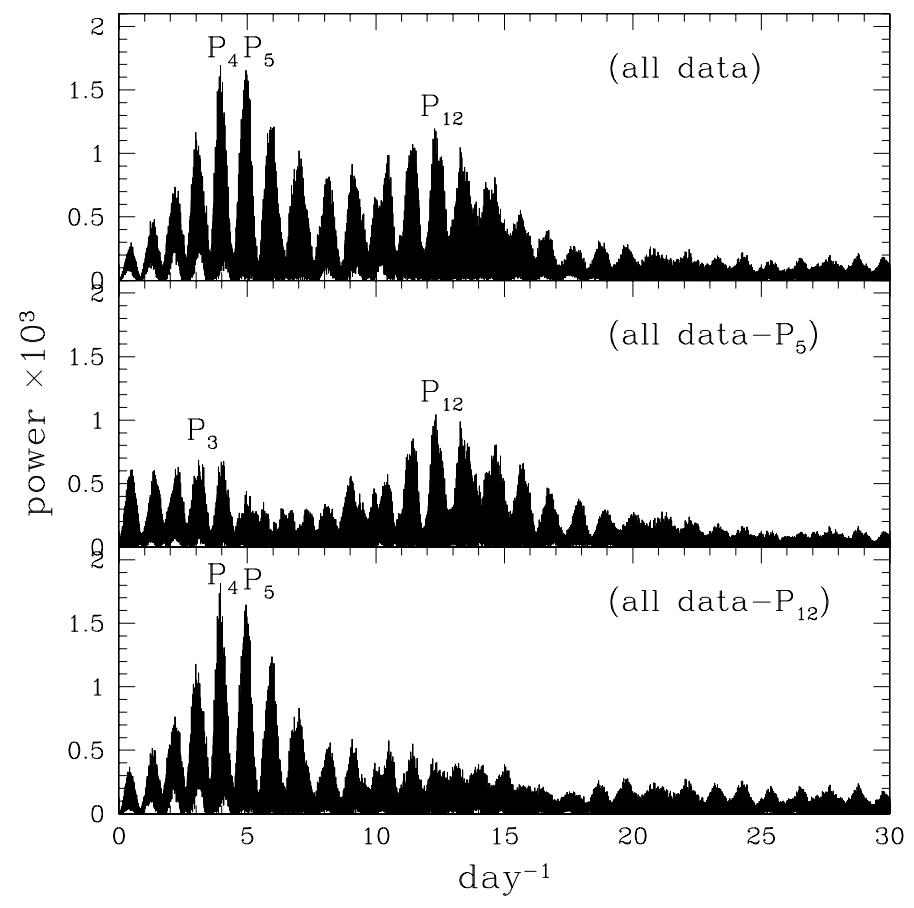

Fig. 3. Upper panel: the power spectra of all the runs obtained with WIRO and Ekar telescopes after subtraction of long-term trends. The two main peaks, $P_{5}$ (which has the same power as its one-day alias $P_{4}$ ) and $P_{12}$, around frequencies $5 \mathrm{~d}^{-1}$ and $12 \mathrm{~d}^{-1}$, respectively, might be correlated with frequency $17 \mathrm{~d}^{-1}$ which corresponds to the historical superhump period PRL. Middle panel: the power spectrum of all the data after subtractions of component $P_{5}$ still shows component $P_{12}$. Bottom panel: the power spectrum of all the data after subtractions of component $P_{12}$ still shows component $P_{5}$.

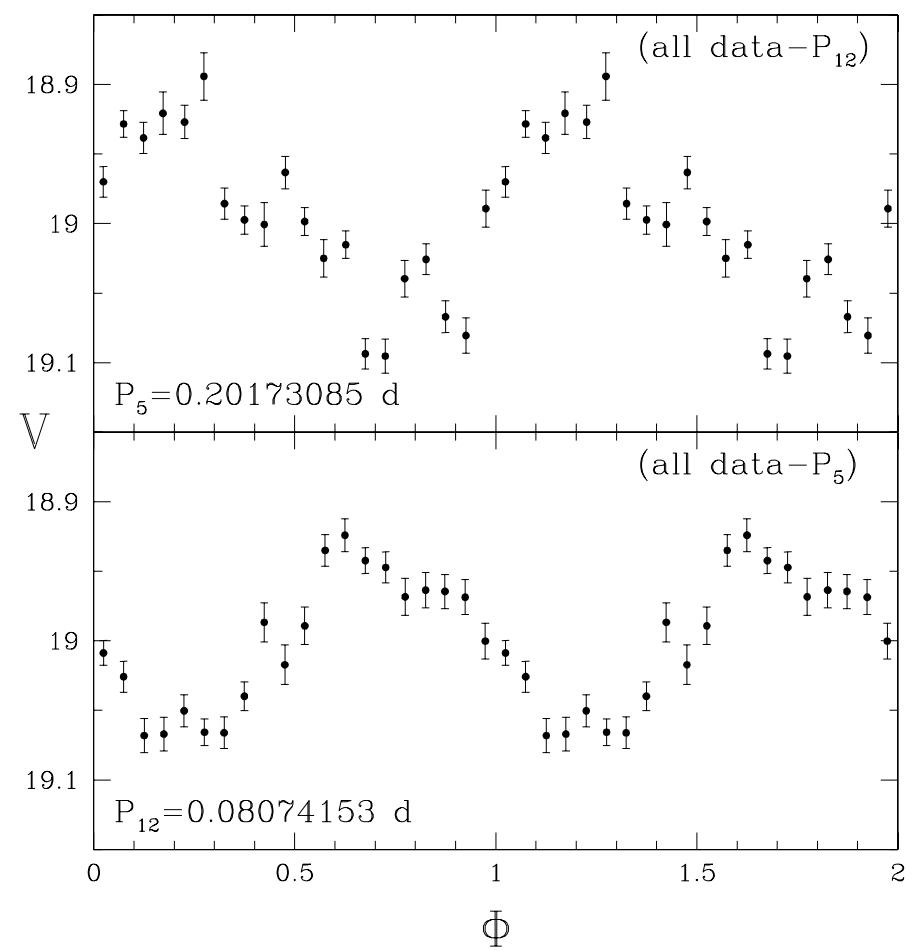

Fig. 4. Upper panel: means of the magnitudes after subtraction of frequency $P_{12}$ and folded with the period corresponding to frequency $P_{5}$. Lower panel: means of the magnitudes after subtraction of frequency $P_{5}$ and folded with the period corresponding to frequency $P_{12}$. The modulation at frequency $P_{12}$ looks better defined. 


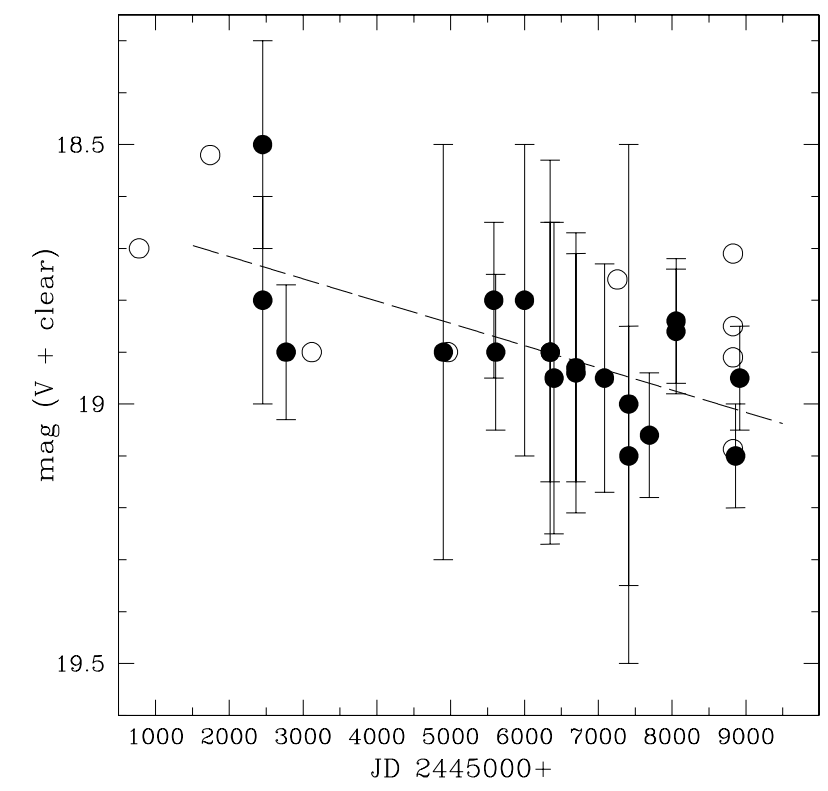

Fig. 5. The long-term photometric trend of the post-nova. Filled circles represent the mean magnitudes of the photometric runs and vertical bars the maximum amplitude of the oscillations observed. Open circles refer to single magnitude determinations. The decline of the old nova is shown by the dashed line that interpolates the filled circles.

of the old nova. Unfortunately, QPOs in CVs are poorly studied because they are contaminated by stochastic flickering (Bruch 1992) and because they often have time scales comparable to the orbital period. The new 2003-2006 time-resolved photometric observations of the old nova show light oscillations with time scales that vary from run to run. Harmonic analysis of all our historical runs demonstrate that these short time-lived oscillations tend to cluster around frequencies $\sim 5$ and $\sim 12 \mathrm{~d}^{-1}$. No power is instead found at the superhump period found by RL. Although the two peak frequencies look correlated with the orbital motion, none of them seems to be the beat between the other one and the orbital period.

The broadness of the two maxima of the power spectrum confirms the QPO nature of the oscillations. In Paper I we mentioned two mechanisms that can produce rather irregular QPOs in CVs. One corresponds to radial and non-radial pulsations of the inner regions of the accretion disk as discussed by Warner (1995). In particular, the superposition of the oscillations of individual disk annuli should produce a range of frequencies from 8 to $40 \times P_{\text {in }}$, with $P_{\text {in }}$ being the Keplerian period of the inner disk radius $r_{\text {in }}$. Thus, an intermediate polar CV could easily present frequencies in the range $3-15 \mathrm{~d}^{-1}$ as RW UMi. It is not clear however what the dominant frequency should be and how it should eventually evolve with time. The second mechanism is based on the vertical thickening of the disk caused by a travelling wave excited in the region immediately inside the corotation radius by the magnetosphere of an inclined magnetic WD rotator. This wave should quickly damp and be regenerated on time-scales comparable to the orbital period (Warner \& Woudt 2002). Thus, this mechanism might be responsible for the higher frequency maximum $P_{12}$ of the all-data power spectrum, as it is closer to the superhump frequency than $P_{5}$.

The post outburst luminosity of RW UMi is still $\sim 2 \mathrm{mag}$ brighter than before the explosion, but it is declining at a rate of $\sim 0.03 \mathrm{mag}^{-1}$. If RW UMi is an intermediate polar, the decrease of $\dot{M}_{2}$ should produce an increase of the magnetospheric radius of the accreting white dwarf primary. The efficiency of the mechanism which can excite the travelling waves responsible for QPOs of the order of the orbital period is maximum in equilibrium accretion conditions, i.e. when the co-rotation radius is slightly larger that the Alfvèn radius. Since RW UMi will reach an equilibrium accretion state only when it comes closer to its quiescent state, the time scales and the amplitudes of the lower frequency QPOs might change with time. Thus, an effort should be made to plan long runs of the old nova which are necessary to detect and analyse in some details low-frequency QPOs and their possible connection with superhump PRL. Future studies will also provide additional information about the mechanism responsible for the light decline.

Acknowledgements. We acknowledge an anonymous referee for helpful comments and suggestions. A.B. wishes to thank the people of the Department of Physics and Astronomy of the University of Wyoming for their friendship and support. This work has been supported by NSF REU Grant AST 9732039, and by the Italian MURST.

\section{References}

Bianchini, A., Tappert, C., Canterna, R., et al. 2003, PASP, 115, 811 (Paper I) Bruch, A. 1992, A\&A, 266, 237

Deeming, T. J. 1975, Astrophys. Space Sci., 36, 137

Duerbeck, H. W. 1987, Space Sci. Rev., 45, 1

Kukarkin, B. V. 1962, IBVS, 18

Patterson, J., \& Warner, B. 1998, PASP, 110, 1026

Retter, A., Leibowitz, E. M., \& Ofek, E. O. 1997, MNRAS, 286, 754

Retter, A., \& Lipkin, Y. 2001, A\&A, 365, 508

Schreiber, M. R., \& Gänsicke, B. 2001, A\&AS, 375, 937

Warner, B. 1995, Cataclysmic Variable Stars (Cambridge: Cambridge Univ. Press)

Warner, B., \& Woudt, P. A. 2002, MNRAS, 335, 84 